\title{
Divergent responses to kisspeptin in children with delayed puberty
}

\author{
Yee-Ming Chan, ${ }^{1,2}$ Margaret F. Lippincott, ${ }^{1}$ Temitope O. Kusa, ${ }^{1}$ and Stephanie B. Seminara ${ }^{1}$ \\ ${ }^{1}$ Harvard Reproductive Sciences Center and Reproductive Endocrine Unit, Massachusetts General Hospital, Boston, \\ Massachusetts, USA. ${ }^{2}$ Division of Endocrinology, Department of Medicine, Boston Children's Hospital, Boston, \\ Massachusetts, USA.
}

BACKCROUND. The neuropeptide kisspeptin stimulates luteinizing hormone (LH) secretion in healthy adults but not in adults with idiopathic hypogonadotropic hypogonadism. We hypothesized that, in children presenting with delayed or stalled puberty, kisspeptin would elicit LH secretion in those children found on detailed nighttime neuroendocrine profiling to have evidence of emerging reproductive endocrine function.

METHODS. Eleven boys and four girls were admitted overnight to assess LH secretion at baseline, after a single intravenous bolus of kisspeptin, and after a single intravenous bolus of gonadotropinreleasing hormone (GnRH). Subjects then received exogenous pulsatile GnRH for 6 days and returned for a second visit to measure responses to kisspeptin and GnRH after this pituitary "priming." Responses to kisspeptin and GnRH were also measured in 5 healthy men.

RESULTS. Of the 15 children with delayed/stalled puberty, 6 exhibited at least one spontaneous LH pulse overnight; all of these subjects had clear responses to kisspeptin, as did one additional subject. Seven subjects had no response to kisspeptin, and one subject exhibited an intermediate response. In the children who responded to kisspeptin, the responses had features comparable to those of adult men.

CONCLUSION. In this first report of kisspeptin administration to pediatric subjects to our knowledge, children with delayed/stalled puberty showed a wide range of responses, with some showing a robust response and others showing little to no response. Further follow-up will determine whether responses to kisspeptin predict future pubertal entry for children with delayed puberty.

TRIAL RECISTRATION. ClinicalTrials.gov NCT01438034 and NCT01952782.

FUNDING. NIH Eunice Kennedy Shriver National Institute of Child Health and Human Development (R01 HD043341, R01 HD090071, P50 HD028138), NIH National Center for Advancing Translational (UL1 TR001102), NIH National Institute of Diabetes and Digestive and Kidney Diseases (T32 DK007028), the Massachusetts General Hospital Executive Committee on Research Fund for Medical Discovery, Harvard Catalyst, Doris Duke Charitable Foundation (award 2013110), Charles H. Hood Foundation, Robert and Laura Reynolds MGH Research Scholar Program, and Harvard University. These funding sources had no role in the design of this study and did not have any role in conducting the study, analyses, interpretation of the data, or the decision to submit results.

Conflict of interest: YMC was a member of the scientific advisory board for AbbVie.

Submitted: December 11, 2017

Accepted: March 8, 2018

Published: April 19, 2018

Reference information:

JCI Insight. 2018;3(8):e99109. https:// doi.org/10.1172/jci.insight.99109.

\section{Introduction}

The neuropeptide kisspeptin sets the reproductive endocrine cascade in motion by stimulating the release of gonadotropin-releasing hormone $(\mathrm{GnRH})$ from the hypothalamus (1). GnRH stimulates secretion of the gonadotropins follicle-stimulating hormone (FSH) and luteinizing hormone ( $\mathrm{LH})$ from the pituitary gland and the gonadotropins in turn stimulate the release of sex steroids and other hormones from the gonads.

The ability of kisspeptin to stimulate GnRH release allows it to be used as the first available probe of human GnRH neuronal activity. Our group and others have demonstrated that administering an intravenous bolus of kisspeptin to healthy men elicits an immediate pulse of LH secretion (2-4). Healthy women in the late follicular and luteal phases of the menstrual cycle similarly respond to kisspeptin with an $\mathrm{LH}$ pulse, though the response is attenuated in the early follicular phase (5-8). 


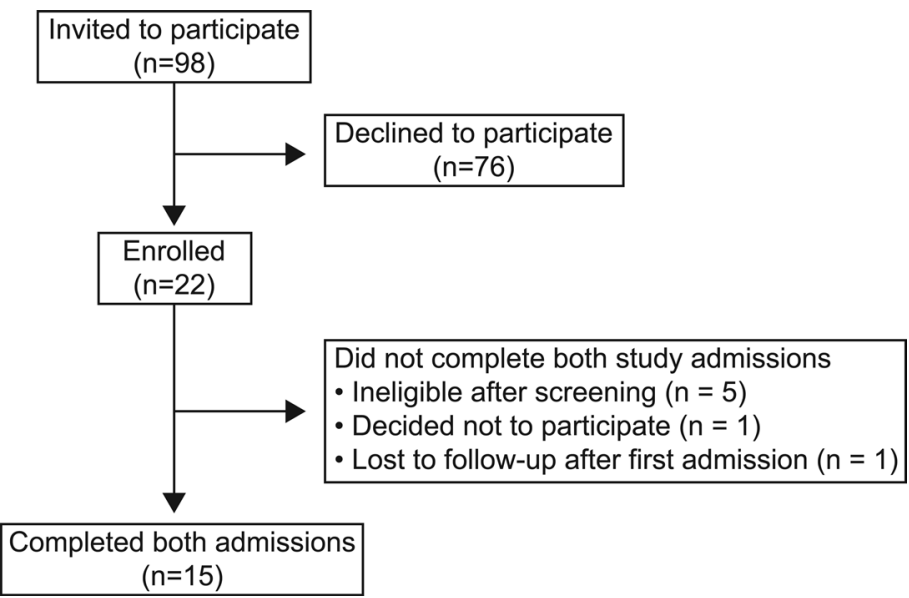

Figure 1. Summary of recruitment.
In contrast, adults with idiopathic hypogonadotropic hypogonadism $(\mathrm{IHH})$, a reproductive endocrine disorder caused by defective secretion or action of $\mathrm{GnRH}$, do not respond to physiologic boluses of kisspeptin $(9,10)$. This failure to respond to kisspeptin was observed even in patients with evidence of GnRH secretion that, while insufficient to achieve normal adult reproductive endocrine activity, was sufficient to induce measurable LH secretion and, in some cases, partial pubertal development. Thus, kisspeptin can be used as a physiologic probe of the ability of the GnRH neuronal network to sustain normal reproductive endocrine activity.

The ability to use kisspeptin to probe GnRH neuronal function presents a potential solution to a frequently encountered clinical challenge: the child presenting with delayed puberty (11). While a small proportion of children presenting with delayed puberty have $\mathrm{IHH}$, the most common cause of delayed puberty is constitutional delay, a self-limited condition in which puberty starts late but progresses normally. The challenge lies in distinguishing between these two diagnoses. Currently, there is no method to predict whether a given patient will eventually enter puberty; a definitive diagnosis can only be made retrospectively, when the patient is either found to enter and eventually complete puberty or not.

A number of tests have been proposed for distinguishing constitutional delay from $\mathrm{IHH}$, but none is perfectly sensitive or specific (12). A common feature of all these tests is that they attempt to detect early hormonal changes associated with the onset of puberty. One of the earliest hormonal changes is the emergence of detectable nighttime, sleep-associated LH pulses, which appear a year or more before physical signs of puberty appear $(13,14)$. However, none of these tests assesses future reproductive endocrine potential, and these tests would all produce negative results in a child with normal pubertal timing if performed well before the onset of puberty. In addition, because currently available tests assess for hormonal signs of very early pubertal development, they are unable to distinguish between constitutional delay and IHH with partial pubertal development $(15,16)$.

In this study, we sought to determine whether, unlike adult cohorts that showed either uniform responsiveness to kisspeptin (healthy adults) or uniform lack of responsiveness (adults with IHH), boys and girls presenting with delayed or stalled puberty would exhibit divergent responses to kisspeptin. Specifically, we hypothesized that children exhibiting nighttime LH pulses (indicating that they are on the cusp of developing physical signs of puberty) would have robust responses to kisspeptin. In addition, because prior work in the rhesus monkey demonstrated that responsiveness to kisspeptin increases with the onset of puberty (17), we compared the magnitude of responses to kisspeptin between children and adults.

\section{Results}

Baseline characteristics. Fifteen subjects, 4 girls and 11 boys, completed the study protocol (Figure 1); characteristics of the subjects are summarized in Table 1. The 4 girls and 8 of the boys were prepubertal on physical examination. The remaining 3 boys presented with stalled pubertal development, with no increase in testicular volume for 6 months or longer. All subjects tolerated study procedures well; there were no major adverse events and no changes in physical examination findings or laboratory results on follow-up.

Study protocol. Subjects came for two visits at the Massachusetts General Hospital (MGH) Clinical Research Center (CRC) (Figure 2). During the first visit, subjects had blood drawn every 10 minutes to assess spontaneous LH pulsatility from 2 am to $8 \mathrm{am}$, then to measure the change in LH in response to kisspeptin $\left(\Delta \mathrm{LH}_{\text {kisspeptin }}\right)$, and then to measure the change in $\mathrm{LH}$ in response to $\mathrm{GnRH}\left(\Delta \mathrm{LH}_{\mathrm{GnRH}}\right)$.

Because GnRH is not easily measurable, kisspeptin-induced $L H$ secretion was used as a surrogate measure of kisspeptin-induced $G n R H$ secretion. However, in prepubertal children with minimal endogenous $\mathrm{GnRH}$ secretion, pituitary responses to GnRH may be attenuated (12). To ensure that endogenous GnRH secretion could be detected, all subjects received pulsatile, exogenous $\mathrm{GnRH}$ for 6 days, which enhances pituitary responsiveness to $\mathrm{GnRH}$, and then returned for a second visit to measure responses to kisspeptin and GnRH after this pituitary "priming." Based on the responses to kisspeptin after priming, we could classify most subjects as either kisspeptin responders or kisspeptin nonresponders. 
Table 1. Baseline characteristics for pediatric subjects

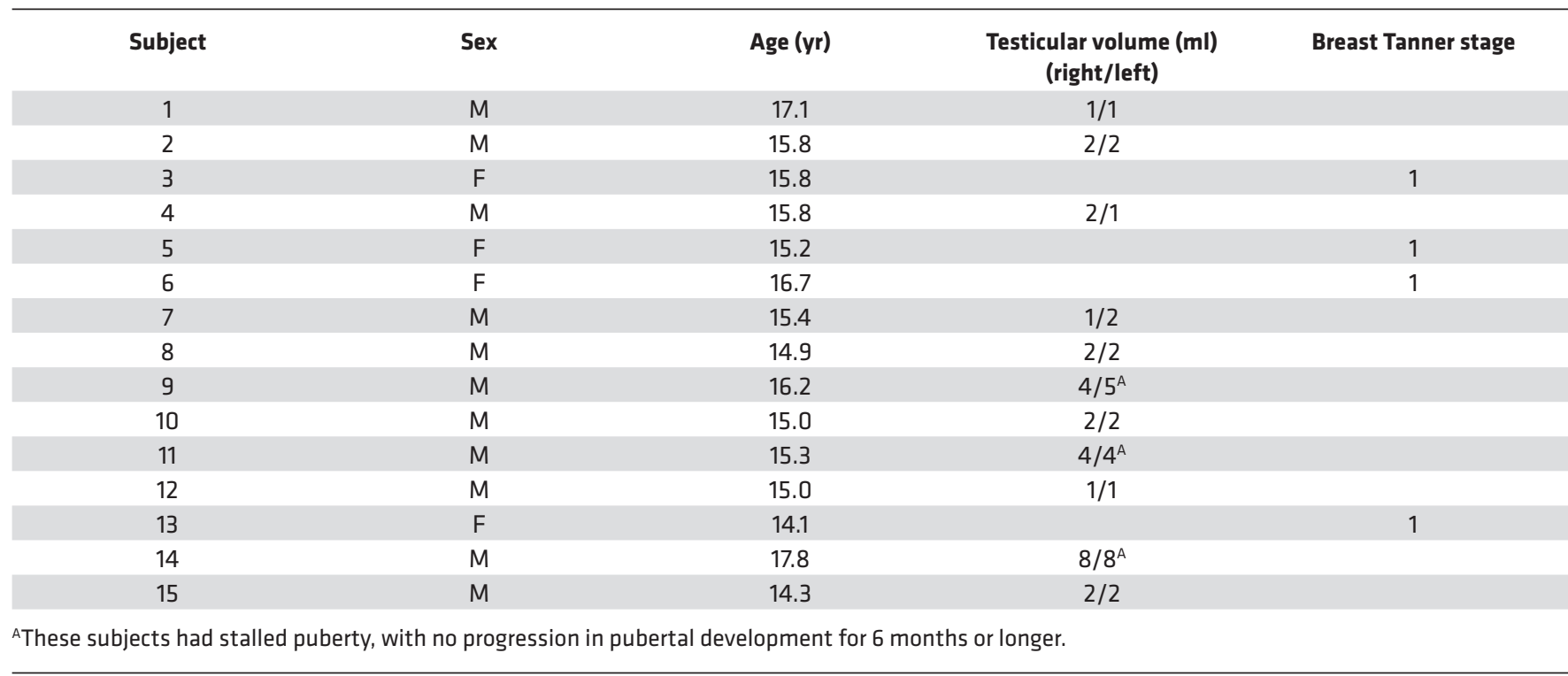

Kisspeptin responders. Seven subjects (6 boys and 1 girl) responded to exogenous kisspeptin with an $\mathrm{LH}$ pulse that met pulse criteria, with a $\Delta \mathrm{LH}_{\text {kispeptin }}$ after priming of $1.2 \pm 0.4 \mathrm{mIU} / \mathrm{ml}$ (mean $\pm \mathrm{SD}$; range 0.8-1.7 mIU/ml; Figure 3A and Supplemental Figure 1; supplemental material available online with this article; https://doi.org/10.1172/jci.insight.99109DS1). Six of these seven subjects also exhibited one or more spontaneous LH pulses on frequent sampling between 2 am and $8 \mathrm{am}$, indicating the presence of endogenous reproductive endocrine activity in these subjects, even though 4 of the subjects appeared prepubertal on physical examination with Tanner I breasts or testicular volumes ranging from 1 to $2 \mathrm{ml}$ (the other 3 presented with stalled pubertal development).

Also consistent with the presence of endogenous reproductive

\section{Admission 1}

(Before priming)

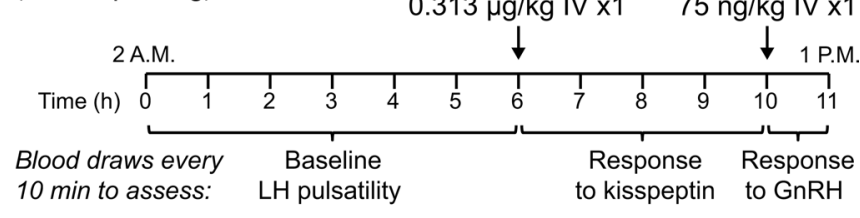

Priming

\section{Admission 2} (After priming)

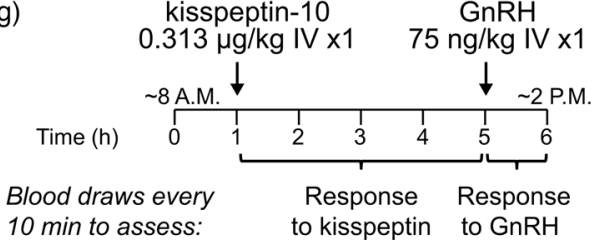

Figure 2. Protocol schematic. Subjects underwent two visits to the Clinical Research Center. During the first visit, blood was drawn every 10 minutes to measure luteinizing hormone (LH) at baseline (from 2-8 am), after an intravenous bolus of kisspeptin, and after an intravenous bolus of gonadotropin-releasing hormone ( $\mathrm{GnRH})$. Subjects then underwent pituitary "priming" with exogenous $\mathrm{GnRH}$ and then returned for a second visit to measure LH after boluses of kisspeptin and GnRH. IV, intravenous; SC, subcutaneous; q2h, every 2 hours. endocrine activity, all responders had a robust response to exogenous $\mathrm{GnRH}$, even before priming (mean $\pm \mathrm{SD} \Delta \mathrm{LH}_{\mathrm{GnRH}} 5.0 \pm 4.8$, range 1.7-15.4 mIU/ml; Table 2, Figure 3A and Supplemental Figure 1). This indicates that endogenous GnRH secretion had already "primed" the pituitary gland in these subjects, and, indeed, further pituitary priming with exogenous $\mathrm{GnRH}$ resulted in little to no increase in $\Delta \mathrm{LH}_{\mathrm{GnRH}}$, with a difference between the $\Delta \mathrm{LH}_{\mathrm{GnRH}}$ before and after priming of -2.4 $\pm 5.0 \mathrm{mIU} / \mathrm{ml}$ (mean $\pm \mathrm{SD}$; range -13.0 to $+0.6 \mathrm{mIU} / \mathrm{ml}, P=0.3$ ).

One boy (subject 11) had a robust response to kisspeptin but did not show LH pulses at baseline (Figure 3B). This subject had received a testosterone cypionate injection 2 days before his visit (as the protocol was designed not to interfere with clinical care, and subjects were allowed to receive sex-steroid treatment while participating in this study). As a result, his serum testosterone was supraphysiologic $(1,179-1,360 \mathrm{ng} / \mathrm{dl})$ at the time of his visit, likely causing suppression of endogenous LH pulsatility. Of note, this subject was able to mount a robust response to kisspeptin $\left(\Delta \mathrm{LH}_{\text {kisspeptin }}\right.$ $1.7 \mathrm{mIU} / \mathrm{ml}$ ) despite his high serum testosterone. At his second visit, serum testosterone had decreased to an early pubertal range $(45-49 \mathrm{ng} / \mathrm{dl})$ and his responses to kisspeptin $\left(\Delta \mathrm{LH}_{\text {kisspeptin }}\right.$ $1.0 \mathrm{mIU} / \mathrm{ml}$ ) and $\mathrm{GnRH}$ remained robust; our protocol does not assess overnight $\mathrm{LH}$ at the second visit, so it is unknown whether he would have exhibited nighttime LH pulses with his lower serum testosterone. 
Table 2. Hormone measurements at baseline, in response to kisspeptin, and in response to GnRH, before and after priming with exogenous GnRH

\begin{tabular}{|c|c|c|c|c|c|c|c|c|c|c|c|c|c|c|}
\hline \multirow[b]{2}{*}{$I D^{A}$} & \multirow[b]{2}{*}{ Sex } & \multicolumn{5}{|c|}{ Before priming } & \multicolumn{8}{|c|}{ After priming } \\
\hline & & $\begin{array}{c}\text { Testosterone } \\
(\mathrm{ng} / \mathrm{dl})\end{array}$ & $\begin{array}{c}\text { Estradiol } \\
(\mathrm{pg} / \mathrm{ml})\end{array}$ & \begin{tabular}{|c|}
$\mathrm{FSH}(\mathrm{mlU} /$ \\
$\mathrm{ml})$
\end{tabular} & $\begin{array}{c}\text { LH } \\
\text { pulses/6 } \\
\text { hours } \\
\end{array}$ & \begin{tabular}{|l|}
$\Delta \mathrm{LH}_{\text {kisspeptin }}$ \\
$(\mathrm{mlU} / \mathrm{ml})$
\end{tabular} & $\begin{array}{c}\Delta \mathrm{LH}_{\mathrm{GnRH}} \\
(\mathrm{mlU} / \mathrm{ml})\end{array}$ & $\begin{array}{c}\Delta \mathrm{LH}_{\text {kisspeptin }} / \\
\Delta \mathrm{LH}_{\text {GnRH }} \\
\text { ratio }\end{array}$ & $\begin{array}{c}\text { Testosterone } \\
(\mathrm{ng} / \mathrm{dl})\end{array}$ & \begin{tabular}{|c|} 
Estradiol \\
$(\mathrm{pg} / \mathrm{ml})$
\end{tabular} & $\begin{array}{c}\mathrm{FSH}(\mathrm{mlU} / \\
\mathrm{ml})\end{array}$ & \begin{tabular}{|c|}
$\Delta \mathrm{LH}_{\text {kisspeptin }}$ \\
$(\mathrm{mlU} / \mathrm{ml})$
\end{tabular} & $\begin{array}{c}\Delta \mathrm{LH}_{\mathrm{EnRH}} \\
(\mathrm{mlU} / \mathrm{ml})\end{array}$ & $\begin{array}{c}\Delta \mathrm{LH}_{\text {kisspeptin }} / \\
\Delta \mathrm{LH}_{\mathrm{GnRH}} \\
\text { ratio }\end{array}$ \\
\hline 1 & M & 25 & & 0.1 & 0 & 0 & 0.2 & 0 & 19 & & 0.9 & 0 & 4.1 & 0 \\
\hline 2 & M & $153^{\mathrm{B}}$ & & $<0.2$ & 0 & 0 & 0.2 & 0 & 16 & & 1.0 & 0 & 7.5 & 0 \\
\hline 3 & $\mathrm{~F}$ & & 4.5 & 0.2 & 0 & 0 & 0.5 & 0 & & 3.5 & 1.0 & 0 & 4.9 & 0 \\
\hline 6 & $\mathrm{~F}$ & & $<2.5$ & 0.5 & 0 & 0 & 1.3 & 0 & & 3.4 & 1.1 & 0 & 7.2 & 0 \\
\hline 7 & $M$ & 4 & & 0.4 & 0 & 0 & 1.6 & 0 & 8.3 & & 2.4 & 0.1 & 15.2 & 0.01 \\
\hline \multicolumn{4}{|c|}{ Mean \pm SD for nonresponders } & & & & $0.7 \pm 0.5$ & & & & & & $6.9 \pm 4.0$ & \\
\hline \multicolumn{15}{|c|}{ Intermediate response to kisspeptin } \\
\hline 8 & M & 12 & & 1.2 & 0 & 0 & 7.5 & 0 & 15 & & 2.2 & 0.4 & 10.4 & 0.04 \\
\hline \multicolumn{15}{|c|}{ Robust responses to kisspeptin } \\
\hline 13 & $\mathrm{~F}$ & & $<2.5$ & 2.5 & 1 & 1.8 & 1.7 & 1.06 & & $<2.5$ & 2.5 & 1.4 & 1.4 & 1.00 \\
\hline 14 & $M$ & $667^{\mathrm{B}}$ & & 0.5 & 2 & 5.4 & 15.4 & 0.35 & 796 & & $<0.2$ & 1.5 & 2.4 & 0.63 \\
\hline 15 & $M$ & 7 & & 5.6 & 3 & 1.5 & 2.0 & 0.75 & 4 & & 3.5 & 1.7 & 1.8 & 0.99 \\
\hline \multicolumn{4}{|c|}{ Mean \pm SD for responders } & & & $2.0 \pm 1.6$ & $5.0 \pm 4.8$ & $0.50 \pm 0.35$ & & & & $1.2 \pm 0.4$ & $2.7 \pm 1.0$ & $0.57 \pm 0.33$ \\
\hline
\end{tabular}

A Listed in order of increasing $\Delta \mathrm{LH}_{\text {kisspeptin }}$ after priming, then increasing $\Delta \mathrm{LH}_{\text {kisspeptin }} / \Delta \mathrm{LH}_{\mathrm{GnRH}}$ ratio after priming, and then increasing $\Delta \mathrm{LH} \mathrm{GnRH}$ before priming. ${ }^{B}$ Measured while treated with exogenous testosterone. $\Delta \mathrm{LH}_{\text {kisspeptin }}$, increase in luteinizing hormone (LH) after kisspeptin; $\Delta \mathrm{LH} \mathrm{GnRH}$, increase in $\mathrm{LH}$ after gonadotropin-releasing hormone (GnRH); $\Delta \mathrm{LH}_{\text {kisspeptin }} / \Delta \mathrm{LH}_{\mathrm{GnRH}}$ ratio, ratio of $\Delta \mathrm{LH}_{\text {kisspeptin }}$ to $\Delta \mathrm{LH}_{\mathrm{GnRH}} ; \mathrm{ND}$, not determined; $\mathrm{M}$, male; $\mathrm{F}$, female.

In addition to having robust responses to kisspeptin after priming, all kisspeptin responders also had clear responses to kisspeptin before priming with the exception of subject 9 (Table 2 and Supplemental Figure 1). He exhibited robust endogenous LH pulsatility that extended into the daytime, and these pulses may have made it difficult to discern his response to kisspeptin before priming. Alternatively, technical problems at the visit before priming, such as improper preparation or administration of kisspeptin, cannot be excluded.

Kisspeptin nonresponders. Four boys and three girls failed to have a significant rise in LH after kisspeptin $\left(\Delta \mathrm{LH}_{\text {kisspeptin }}\right.$ after priming $=0 \mathrm{mIU} / \mathrm{ml}$ in 6 subjects, $0.1 \mathrm{mIU} / \mathrm{ml}$ in the seventh $)$; all of these subjects presented with no signs of puberty, with Tanner I breasts or testicular volumes ranging from 1 to $2 \mathrm{ml}$ (Figure $4 \mathrm{~A}$ and Supplemental Figure 1). Baseline LH in these subjects was near or below the limits of assay detection, and they had no detectable endogenous LH pulses. Furthermore, responses to GnRH before priming were small $(\Delta \mathrm{LH}-$ GnRH $0.2-1.3 \mathrm{mIU} / \mathrm{ml})$ and increased significantly after priming with exogenous pulsatile $\mathrm{GnRH}\left(\Delta \mathrm{LH}_{\mathrm{GnRH}}\right.$ after priming 3.3-7.5 $\mathrm{mIU} / \mathrm{ml}$, difference between $\Delta \mathrm{LH}_{\mathrm{GnRH}}$ before and after priming $6.2 \pm 1.6 \mathrm{mIU} / \mathrm{ml}$, range +2.7 to $+13.6 \mathrm{mIU} / \mathrm{ml}, P<0.001$; Table 2), demonstrating that the cause of the small responses to $\mathrm{GnRH}$ before priming was lack of prior exposure to $\mathrm{GnRH}$, i.e., the lack of robust endogenous $\mathrm{GnRH}$ secretion.

Intermediate responder. An intermediate response to kisspeptin $\left(\Delta \mathrm{LH}_{\text {kisspeptin }}\right.$ after priming $\left.0.4 \mathrm{mIU} / \mathrm{ml}\right)$ was observed in one boy who presented with no signs of puberty (subject 8 and Figure 4B). His baseline LH was measurable but apulsatile (ranging from $0.4-0.5 \mathrm{mIU} / \mathrm{ml}$ ), suggesting the presence of some endogenous $\mathrm{GnRH}$ secretion. Consistent with this, he had a robust response to $\mathrm{GnRH}$ before priming $\left(\Delta \mathrm{LH}_{\mathrm{GnRH}} 7.5 \mathrm{mIU} /\right.$ $\mathrm{ml}$ ). Of note, his response to kisspeptin was discernible only after pituitary priming.

GnRH neuronal responses to kisspeptin in children compared with adults. To compare responses to kisspeptin between children and adults, we assessed LH responses to kisspeptin and GnRH in 5 healthy adult men (Table 3). The kisspeptin-induced LH pulse amplitude in adult men was $4.9 \pm 2.0 \mathrm{mIU} / \mathrm{ml}(\mathrm{mean} \pm \mathrm{SD}$ ), significantly greater than what was observed in children $(0.6 \pm 0.7 \mathrm{mIU} / \mathrm{ml}, P=0.008$; Table 2$)$. This difference was seen even if the analysis was restricted to the 7 children who had robust responses to kisspeptin $\left(\Delta \mathrm{LH}_{\text {kisspeptin }} 1.2 \pm 0.4 \mathrm{mIU} / \mathrm{ml}, P=0.013\right.$; Table 2$)$. 


\section{A} Subject 13
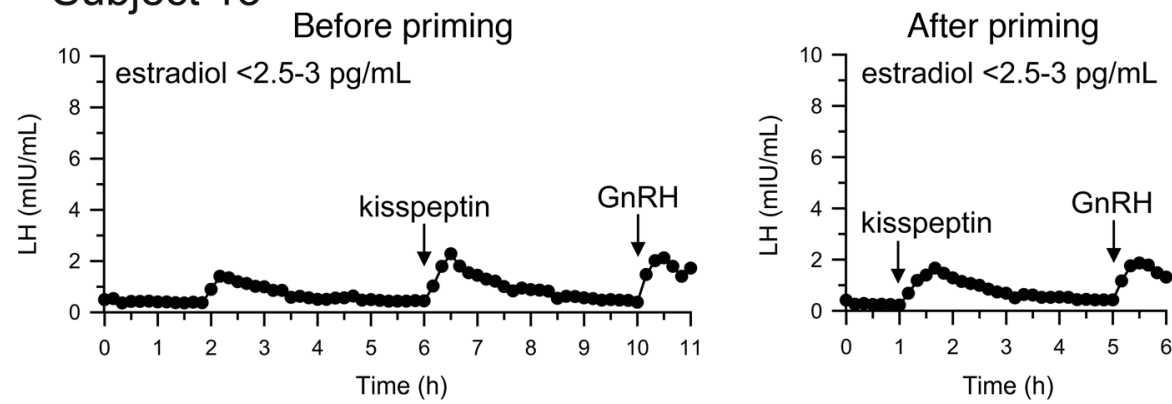

\section{B Subject 11}
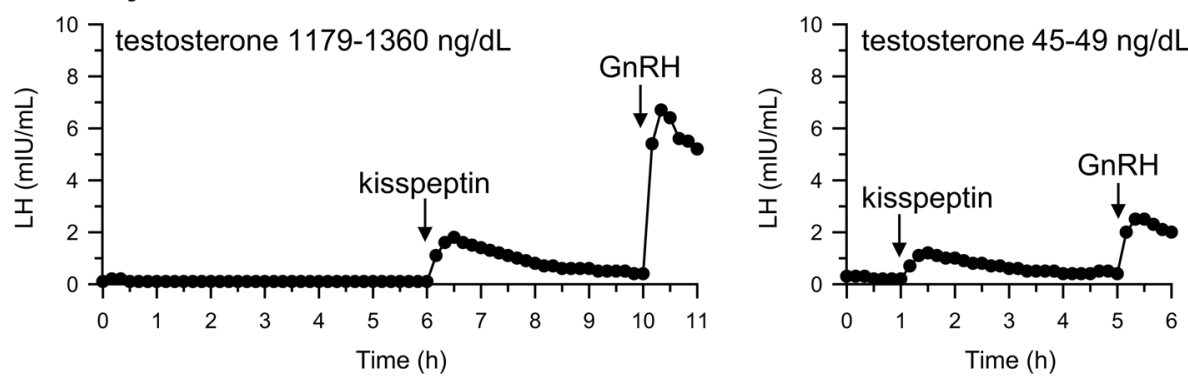

Figure 3. Neuroendocrine profiles of subjects with robust responses to kisspeptin. Serum luteinizing hormone (LH) concentrations were measured at baseline (hours 0-6), after exogenous kisspeptin (hours 6-10), and after exogenous gonadotropin-releasing hormone (GnRH; hours 10-11). Subjects returned after priming with exogenous $\mathrm{GnRH}$, and serum LH was measured at baseline (hours 0-1), after exogenous kisspeptin (hours 1-5), and after exogenous GnRH (hours 5-6). Baseline concentrations of serum sex steroids (estradiol and testosterone) were determined by measuring pools of samples collected between 0 and 2 hours, 2 and 4 hours, and 4 and 6 hours during the visit before priming and of samples collected between 0 and 1 hour in the visit after priming. (A) A representative "responder" (1 of 7) who before priming demonstrated an endogenous LH pulse, a clear response to kisspeptin, and a robust response to $\mathrm{GnRH}$ and after priming demonstrated little change in the responses to kisspeptin and GnRH. (B) A "responder" who did not exhibit baseline LH pulses, most likely because he had recently received a dose of exogenous testosterone and the resulting high serum testosterone suppressed endogenous LH secretion. Note that the response to kisspeptin was still robust.

Because we used LH as a surrogate measure of GnRH secretion, the larger LH responses to kisspeptin observed in adults compared with children could be due to differences in GnRH neuronal responsiveness to kisspeptin, differences in pituitary responsiveness to GnRH, or both. To distinguish among these possibilities, we compared LH responses to exogenous $G n R H$ (Figure 4) and found that $\Delta \mathrm{LH}_{\mathrm{GnRH}}$ was also larger in adults than in children with robust responses to kisspeptin (11.4 \pm 6.2 vs. $2.6 \pm 1.0, P=0.03$; Tables 2 and 3). We then estimated the amount of GnRH secreted in response to kisspeptin by normalizing the LH response to kisspeptin ( $\Delta \mathrm{LH}_{\text {kisspeptin }}$ ) to the $\mathrm{LH}$ response to $G n R H\left(\Delta \mathrm{LH}_{\mathrm{GnRH}}\right)$ and expressed this as a ratio between the two responses ( $\Delta \mathrm{LH}_{\text {kisspeptin }} / \Delta \mathrm{LH}_{\text {GnRH }}$ ratio). The $\Delta \mathrm{LH}_{\text {kisseptin }} / \Delta \mathrm{LH}_{\mathrm{GnRH}}$ ratio after priming in children with robust responses to kisspeptin was $0.57 \pm 0.33$, which was not significantly different from the $\Delta \mathrm{LH}_{\text {kisspeptin }} / \Delta \mathrm{LH}_{\text {GnRH }}$ ratio in adult men $(0.48 \pm 0.17, P=0.6$; Figure 5 and Tables 2 and 3$)$. Thus, while LH responses to kisspeptin were larger in adult men than in children with delayed puberty, this appeared to be entirely due to increased pituitary responsiveness to $\mathrm{GnRH}$, and no difference in GnRH neuronal responsiveness to kisspeptin was observed.

In previous work in adult men, we examined the morphology of kisspeptin-induced LH pulses as an indirect way to assess GnRH neuronal responses to kisspeptin (3). Specifically, by examining the time required for a kisspeptin-induced LH pulse to reach its peak, we inferred that a single intravenous dose of kisspeptin induced a prolonged episode of GnRH secretion, lasting approximately 17 minutes (3). Applying a similar analysis to the data from children with delayed puberty, we observed that the time to peak was significantly longer for kisspeptin-induced LH pulses than for GnRH-induced LH pulses (mean $\pm \mathrm{SD}$, kisspeptin induced $28.3 \pm 4.1 \mathrm{~min} ; \mathrm{GnRH}$ induced $21.7 \pm 4.1 \mathrm{~min}, P=0.03$, Supplemental Figure 2). Of note, the time to peak after kisspeptin and GnRH in children with delayed puberty was similar to what we observed in the above adult men (mean \pm SD, kisspeptin induced 26.0 $\pm 5.5 \mathrm{~min} ; \mathrm{GnRH}$ induced $22.0 \pm 4.5 \mathrm{~min})$. 
A

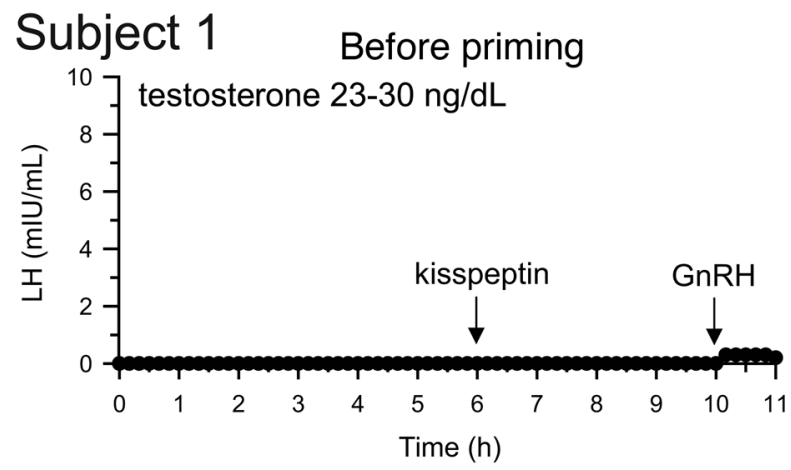

Kisspeptin Non-Responder

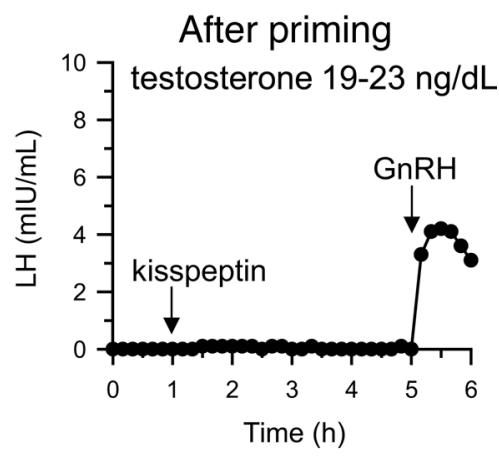

Figure 4. Neuroendocrine profiles of subjects whose responses to kisspeptin were absent or small. (A) A representative "nonresponder" (1 of 7) who demonstrated no endogenous luteinizing hormone (LH) pulses, no response to kisspeptin (before or after priming), and a small response before priming to gonadotropin-releasing hormone $(\mathrm{GnRH})$ that was augmented by priming with repeated doses of exogenous GnRH. (B) An intermediate response to kisspeptin in a subject whose baseline LH was measurable but apulsatile and who had a robust response to exogenous $\mathrm{GnRH}$ even before priming.

B

Subject 8

Kisspeptin Intermediate Responder
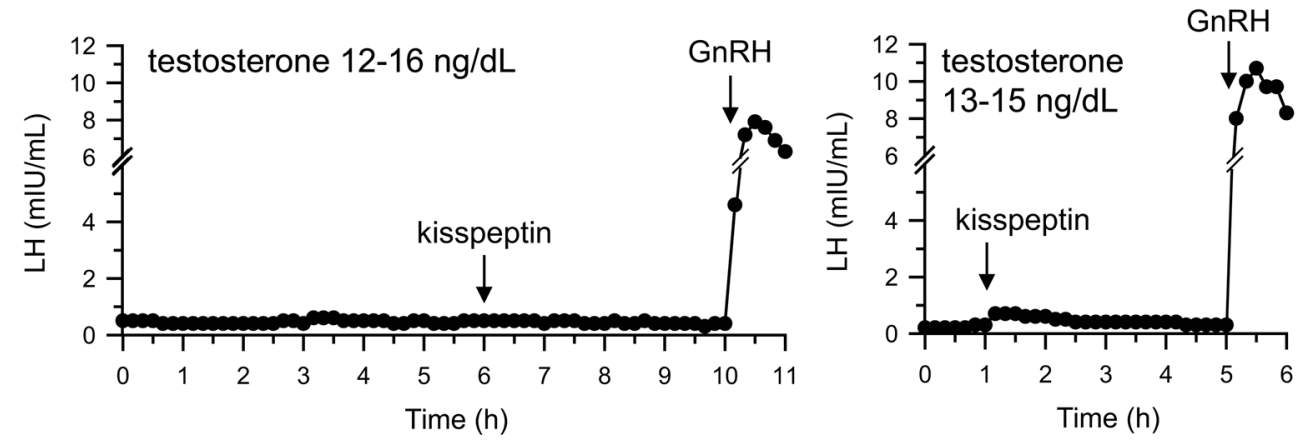

\section{Discussion}

In this first report to our knowledge of kisspeptin administration to pediatric subjects, 15 boys and girls with delayed or stalled puberty underwent detailed neuroendocrine profiling to assess LH secretion at baseline, in response to kisspeptin, and in response to GnRH. We identified 7 kisspeptin responders, 7 nonresponders, and 1 subject with an intermediate response. Our results demonstrate that kisspeptin can elicit reproductive endocrine activity in children who otherwise appear prepubertal on clinical evaluation. Furthermore, the varied neuroendocrine profiles of these children resemble patterns that we and others had previously charted in healthy adults and adults with IHH (2-10). These similarities suggest that the kisspeptin stimulation test has the potential to be a physiologically based method for diagnosing $\mathrm{IHH}$ in childhood, well before the age threshold of 18 years used in the current empirical definition of $\mathrm{IHH}$.

Table 3. Responses to kisspeptin and GnRH in healthy adult men

\begin{tabular}{|c|c|c|c|}
\hline ID $^{\mathrm{A}}$ & $\Delta \mathrm{LH}_{\text {kisspeptin }}(\mathrm{mlU} / \mathrm{ml})$ & $\Delta \mathrm{LH}_{\mathrm{GnRH}}(\mathrm{mlU} / \mathrm{ml})$ & $\Delta \mathrm{LH}_{\text {kisspeptin }} / \Delta \mathrm{LH}_{\mathrm{GnRH}}$ ratio \\
\hline $\mathrm{A} 2$ & 8.1 & 20.6 & 0.39 \\
\hline A4 & 3.5 & 7.1 & 0.49 \\
\hline A5 & 3.3 & 4.3 & 0.77 \\
\hline Mean \pm SD & $4.9 \pm 2.0$ & $11.4 \pm 6.2$ & $0.48 \pm 0.17$ \\
\hline
\end{tabular}

A Listed in order of increasing $\Delta \mathrm{LH}_{\text {kisspeptin }} / \Delta \mathrm{LH}_{\mathrm{GnRH}}$ ratio. $\Delta \mathrm{LH}_{\text {kisspeptin }}$, increase in luteinizing hormone (LH) after kisspeptin; $\Delta \mathrm{LH} \mathrm{GnRH}$, increase in $\mathrm{LH}$ after gonadotropin-releasing hormone (GnRH); $\Delta \mathrm{LH}_{\text {kisspeptin }} / \Delta \mathrm{LH}_{\mathrm{GnRH}}$ ratio, ratio of $\Delta \mathrm{LH}_{\text {kisspeptin }}$ to $\Delta \mathrm{LH}_{\mathrm{GnRH}}$ 


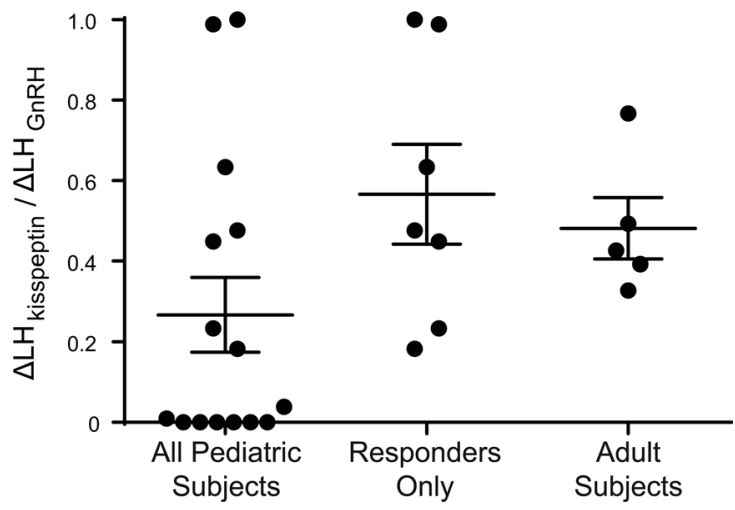

Figure 5. Magnitude of responses to kisspeptin in pediatric subjects and adult subjects. To estimate the response of gonadotropin-releasing hormone (GnRH) neurons to kisspeptin and to account for potential differences in pituitary responsiveness to $\mathrm{GnRH}$, the amplitude of kisspeptin-induced luteinizing hormone (LH) pulses $\left(\Delta \mathrm{LH}_{\text {kisspeptin }}\right)$ was normalized to the amplitude of $\mathrm{GnRH}$-induced LH pulses $\left(\Delta \mathrm{LH}_{\mathrm{GnRH}}\right)$, and the ratios between these two amplitudes $\left(\Delta \mathrm{LH}_{\text {kisspeptin }} / \Delta \mathrm{LH}_{\mathrm{GnRH}}\right)$ are shown. Bars show means and standard deviations. No significant differences were observed between groups with unpaired $t$ tests. $n=15$ total pediatric subjects, 7 responders; $n=5$ adult subjects.

As we hypothesized, all individuals with spontaneous nighttime LH pulses showed robust responses to kisspeptin. The presence of LH pulses indicates that these children were actually peripubertal, despite the fact that some appeared prepubertal on external physical examination (the others presented with stalled pubertal development). All of the responders also had responses to GnRH that were robust even before pituitary priming and were not augmented further by priming with exogenous $\mathrm{GnRH}$, demonstrating that these subjects were secreting GnRH endogenously. The neuroendocrine profiles of these responders are similar to the normal $\mathrm{LH}$ pulsatility and robust responses to kisspeptin that we and others have observed in healthy adult men and in healthy adult women in the late follicular and luteal phases of the menstrual cycle (2-8).

Seven subjects did not respond to kisspeptin. The lack of responses to kisspeptin and other aspects of their neuroendocrine profiles are similar to what we have observed in adults with $\mathrm{IHH}$, a disease characterized by failure to achieve normal reproductive endocrine function by age 18 years due to defects in $\mathrm{GnRH}$ secretion or action (9). In the profiles of both the pediatric nonresponders and the adults with IHH, baseline $\mathrm{LH}$ is low and apulsatile. Furthermore, responses to $\mathrm{GnRH}$ before priming are small but can be augmented by priming with exogenous $\mathrm{GnRH}$; the robust responses to $\mathrm{GnRH}$ after priming demonstrate that the diminutive responses to GnRH before priming were not due to a defect in the pituitary gonadotropes but rather were due to lack of sufficient endogenous GnRH secretion to maintain the pituitary glands in a "primed" state. While we cannot exclude the possibility that kisspeptin responsiveness may increase over time (for instance, if repeated exposure to kisspeptin is necessary for $\mathrm{GnRH}$ neurons to become fully responsive), if the neuroendocrine profiles of the nonresponder children are in fact fixed and persist into adulthood, we anticipate that these children will fail to enter puberty and eventually earn a diagnosis of IHH.

Indeed, several previously proposed ways to distinguish between constitutional delay and IHH were observed as differences between the kisspeptin responders and nonresponders in this study. As noted above, the two groups differed in terms of the presence/absence of endogenous nighttime LH pulses and responses to $\mathrm{GnRH}$ before priming (though the ranges for the latter were nearly overlapping). However, other features proposed to differ between children with CDP and those with $\mathrm{IHH}$ - first morning $\mathrm{LH}$, first morning FSH, and testicular volume (for boys) $(12,18)$ - were not notably different between the responder and nonresponder groups (see Tables 1 and 2). In addition, one subject had a neuroendocrine profile that differed from both the responders and the nonresponders.

This subject (subject 8) exhibited an intermediate response to kisspeptin, and his neuroendocrine profile resembles those seen in a special subset of adults with IHH (9). Though patients with IHH classically present with complete absence of reproductive endocrine activity, as described above, some individuals with IHH have evidence of partial neuroendocrine activity $(9,15,16,19)$. These adults have measurable serum LH, but their LH secretory patterns are often apulsatile. Furthermore, they exhibit robust responses to exogenous $\mathrm{GnRH}$ even before pituitary priming, demonstrating the presence of enough endogenous $\mathrm{GnRH}$ secretion to maintain pituitary responsiveness to $\mathrm{GnRH}$, though not enough to sustain normal reproductive endocrine function. We previously demonstrated that, despite the presence of some endogenous GnRH activity, these adults fail to respond to exogenous kisspeptin at doses that elicit robust responses in reproductively normal adults (9). Like these adults, subject 8 had baseline LH that was measurable but apulsatile and a robust response to GnRH even before priming. However, his prepubertal testicular volumes and low serum testosterone demonstrate that this $\mathrm{GnRH}$ secretion was unable to drive pubertal 
development, possibly because of defective organization or coordination of the GnRH neuronal network, leading to lack of pulsatility; alternatively or in addition, this subject could have deficits in pituitary or testicular function, which have been observed in some individuals with IHH (20). If this subject's diminished response to kisspeptin persists into adulthood, it seems likely that he will be found to have IHH.

Measuring responses to kisspeptin in pediatric subjects provided an opportunity to compare responses to kisspeptin between children and adults. In rhesus macaques, responsiveness of the $\mathrm{GnRH}$ neuronal network to kisspeptin increases with pubertal development, with midpubertal monkeys releasing more $\mathrm{GnRH}$ in response to kisspeptin than prepubertal monkeys (17). In humans, we found that the LH response to kisspeptin was larger in adults than in the peripubertal children showing the "responder" pattern. However, this difference was entirely attributable to greater pituitary responsiveness to GnRH in adults; when we specifically isolated the response of the GnRH neuronal network to kisspeptin (by normalizing the LH response to kisspeptin to the LH response to $G n R H$ ), we observed no difference between adults and children. Taken together, the data comparing prepubertal and midpubertal rhesus monkeys suggest that GnRH neuronal responsiveness to kisspeptin increases across the transition from the prepubertal to the peripubertal state, and our current data comparing peripubertal children to adult men suggest that kisspeptin responsiveness remains constant from the peripubertal period and on. However, we cannot exclude differences between species; for example, the increase in GnRH neuronal responsiveness to kisspeptin across sexual maturation may occur in rhesus monkeys but not in humans. Assessing the responses of prepubertal children to kisspeptin would distinguish between these two possibilities.

In these initial studies of the effects of kisspeptin in pediatric subjects, we used a complex protocol to fully characterize the response to kisspeptin. More widespread use of kisspeptin will require a simpler protocol, and our data have several practical implications for the implementation of kisspeptin stimulation testing in the clinical setting.

Concurrent use of sex steroids. One subject (subject 11) had received an injection of testosterone 2 days prior to his research visit. As a result, his serum testosterone was supraphysiologic and likely suppressed the production of endogenous LH pulses through negative feedback, as he did not exhibit LH pulses at baseline. Nonetheless, he showed a robust response to kisspeptin, despite his high serum testosterone. This finding is consistent with the model in which the negative feedback effects of sex steroids occur at the level of the kisspeptin neuron (which, unlike the GnRH neuron, expresses receptors for estrogens, androgens, and progesterone), such that administration of exogenous kisspeptin could bypass this negative feedback (21). Our findings also demonstrate that the response to kisspeptin can be reliably detected irrespective of circulating sex-steroid concentrations.

Need for pituitary priming. In 6 of the 7 responders, the responses to kisspeptin were observed both before and after pituitary priming, but in the seventh responder (subject 9), the response to kisspeptin was detectable only after pituitary priming with exogenous GnRH. This particular individual exhibited pulsatile LH secretion not only at night but also during the day, such that routine daytime laboratory evaluation would have readily detected the presence of reproductive endocrine activity. Furthermore, the response to kisspeptin in the intermediate responder (subject 8) was seen only after pituitary priming. Why these subjects' kisspeptin responses were observed only after exposure to exogenous GnRH - and whether this reflects a biological action of $\mathrm{GnRH}$ or a merely a technical issue with kisspeptin administration at the first visit - is unclear. Regardless, from a practical perspective, our data suggest that pituitary priming is not required in most individuals for accurately evaluating the LH response to kisspeptin.

Timing of the LH peak after kisspeptin. In the children who responded to kisspeptin, kisspeptin-induced LH pulses took longer to reach their peaks than those induced by GnRH, similar to what we had previously observed in adult men. This finding suggests that kisspeptin induces sustained GnRH release in both peripubertal children and adults and that the dynamics of GnRH neuronal responses to kisspeptin are already established by the peripubertal period. From a practical perspective, the fact that peak LH was reached at or near 30 minutes after kisspeptin administration indicates that a single measurement at the +30 -minute time point would be sufficient to assess a child's response to kisspeptin.

In summary, we have identified distinct responses to kisspeptin and neuroendocrine profiles in children presenting with delayed or stalled puberty: a "responder" profile that resembles that of adults with normal reproductive endocrine function, a "nonresponder" profile that resembles that of adults with $\mathrm{IHH}$ who have no reproductive endocrine function, and an "intermediate responder" profile 
that resembles that of adults with $\mathrm{IHH}$ who have partial reproductive endocrine function. Ongoing follow-up of these subjects to establish their final diagnoses will determine the ability of the kisspeptin stimulation test to predict outcomes for children presenting with delayed or stalled puberty.

\section{Methods}

Hormone sources and preparation. GnRH was synthesized under good manufacturing practices (GMP) and purchased from Polypeptide Laboratories. GMP-grade kisspeptin-10 (corresponding to amino acids $112-121$ of the preprohormone) was also synthesized by Polypeptide Laboratories, under contract to the Eunice Kennedy Shriver National Institute of Child Health and Human Development (NICHD), and was provided as a gift. Resuspended aliquots underwent tests for sterility, pyrogenicity, purity, and concentration.

Pediatric subjects. Pediatric subjects were recruited from 2011 to 2016 from the pediatric endocrinology clinics of Boston Children's Hospital and MGH and were also referred from pediatric endocrinologists across the United States. Inclusion criteria for girls were age $\geq 12$ years and either no palpable breast tissue (absence of pubertal development) or no progression in breast development for 6 months or longer (stalled pubertal development). Inclusion criteria for boys were age $\geq 13.5$ years and testicular volume $<4$ $\mathrm{ml}$, as assessed by Prader orchidometer (absence of pubertal development), or no increase in testicular volume for 6 months or longer (stalled pubertal development). Exclusion criteria were (a) any evidence for a potential underlying cause of delayed/stalled puberty on history (e.g., disordered eating, intense endurance exercise, a medical condition that could affect reproductive endocrine function), physical examination (e.g., BMI <10th percentile for bone age), laboratory evaluation (e.g., positive celiac serology, thyroid dysfunction, elevated erythrocyte sedimentation rate), or imaging studies (hypothalamic/ pituitary abnormality on cranial MRI); (b) history of an allergic drug reaction requiring emergency medical treatment; (c) weight below $28 \mathrm{~kg}$ (to avoid exceeding safety limits for blood draws); and (d) clinically relevant abnormality on complete blood count, serum electrolytes, AST, ALT, TSH, free T4, or IGF-1. No restrictions or requirements were placed on subjects' clinical care; subjects could therefore be treated with exogenous sex steroids while participating in this study.

Pediatric protocol. In this single-arm, open-label study, pediatric subjects underwent two admissions to the MGH CRC (Figure 2). From 2 am to 8 am, subjects had 2-3 $\mathrm{ml}$ of blood drawn every 10 minutes using a blood-sparing protocol to assess overnight baseline LH pulsatility. At 8 am, subjects received a single intravenous bolus of $0.313 \mu \mathrm{g} / \mathrm{kg}$ kisspeptin $(0.24 \mathrm{nmol} / \mathrm{kg})$, the same dose used in our adult studies of kisspeptin $(3,7,9,10)$, and blood continued to be drawn from 8 am to 12 noon to assess the response to kisspeptin. At noon, subjects received a single intravenous bolus of $75 \mathrm{ng} / \mathrm{kg} \mathrm{GnRH}$, and blood was drawn from noon to $1 \mathrm{pm}$ to assess the response to GnRH.

In prepubertal children, the responsiveness of the pituitary gonadotropes to GnRH may be minimal and increases only with repeated exposure to $\mathrm{GnRH}$ ("priming"). Subjects therefore underwent pituitary priming, with GnRH 25 ng/kg delivered subcutaneously by a portable Crono F pump (Canè S.p.A) every 2 hours for 6 days, with the last dose of GnRH given approximately 48 hours prior to the second CRC admission. To ensure the safety of ongoing blood draws, plasma hemoglobin was measured either 1-5 days prior to the second CRC admission or the morning of the second CRC admission.

At the second CRC admission, subjects underwent 1 hour of baseline frequent blood sampling starting around $9 \mathrm{am}$, received an intravenous bolus of $0.313 \mu \mathrm{g} / \mathrm{kg}$ kisspeptin followed by 4 hours of blood sampling, and then received an intravenous bolus of $75 \mathrm{ng} / \mathrm{kg} \mathrm{GnRH}$ followed by 1 hour of blood sampling. A follow-up visit was held 2-4 weeks after the second CRC admission to obtain an interval history, conduct a repeat physical examination, and assess safety laboratory studies.

Adult subjects and protocol. Healthy adult volunteers were recruited through advertisements and met the following criteria: (a) age 21-40 years; (b) no chronic health conditions or medications; (c) normal timing of puberty and normal sexual function by report; (d) normal physical examination; and (e) normal complete blood count, serum electrolytes, AST, ALT, TSH, and free T4. Subjects were admitted to the MGH CRC for 14 hours of blood sampling every 10 minutes. Kisspeptin $(0.313 \mu \mathrm{g} / \mathrm{kg})$ was given at the 6-hour time point, and $75 \mathrm{ng} / \mathrm{kg} \mathrm{GnRH}$ was given at the 12-hour time point.

Laboratory assays. Two assays were used to measure LH for the pediatric studies. For initial studies, LH was measured by the MGH Clinical Laboratory Research Core using the Architect immunoassay (Abbott Laboratories). Midway through the study, the facility closed; for later studies, LH was measured by the 
Brigham and Women's Hospital Research Assay Core using the Access immunoassay (Beckman Coulter). Control samples from prior studies were sent to both laboratories, and results showed good correlation $\left(\mathrm{R}^{2}\right.$ 0.996). For adult studies, LH was measured by the MGH Clinical Laboratory Research Core using the Architect immunoassay (Abbott Laboratories).

A validated modification of the method of Santen and Bardin was used to identify LH pulses $(19,22)$. LH pulse amplitude was calculated as the peak LH in the 1 hour after drug administration subtracted by the LH measurement just prior to drug administration; negative results were converted to 0 . The time to peak was defined as the time that was required for peak LH to be reached after kisspeptin or GnRH administration.

FSH was measured by immunoassay, and estradiol and testosterone were measured by liquid chromatography/tandem mass spectrometry by measuring pooled samples collected between 1 and 2 hours by LabCorp.

Statistics. Data are reported as mean \pm SD. Paired 2-tailed Student's $t$ tests were used to compare responses to $\mathrm{GnRH}$ before and after pituitary priming and to compare time to peak of LH pulses after kisspeptin and after GnRH. Unpaired 2-tailed Student's $t$ tests were used to compare pediatric and adult responses to kisspeptin. GraphPad Prism was used for statistical analyses. $P$ values of less than 0.05 were considered significant.

Study approval. The studies in children with delayed puberty and in adults were approved by the IRB of MGH/Partners Healthcare (protocols 2011P002885 and 2013P001543, respectively) and registered with ClinicalTrials.gov (NCT01438034 and NCT01952782, respectively). GnRH was used under investigational new drug application 93,353, and kisspeptin was used under investigational new drug application 74,978 (for adult men) and application 113,591 (for children). Written informed consent was obtained from all adult subjects and from at least one parent or guardian for pediatric subjects, and assent was obtained from all pediatric subjects.

\section{Author contributions}

YMC, MFL, and SBS designed the research studies. YMC, MFL, and TOK acquired data. YMC and TOK analyzed data. YMC wrote the first draft of the manuscript, which was reviewed by all authors.

\section{Acknowledgments}

We thank the subjects and their families and Michael Agus, Cielo Alleyn, Paul Boepple, Jill Brodsky, Rosalind Brown, Gregory Goodwin, Hector Granados, Eray Savgan-Gurol, and Abhinash Srivatsa for referring subjects. We also thank the staff of the MGH CRC and Dianali Rivera Morales, Voula Christopoulos, Cindy Li, Casey Cokkinias, Johanna Clair, Ryan Ciarlo, Elfa Jonsdottir, Amalia Feld, and other members of the MGH Reproductive Endocrine Unit for their assistance and input. This work was supported by a Harvard Catalyst Pilot Grant (to YMC and SBS), a Doris Duke Charitable Foundation Clinical Scientist Development Award (2013110 to YMC), a Charles H. Hood Foundation Child Health Research Grant (to YMC), and an NIH/NICHD grant (R01 HD043341 to SBS). YMC was further supported by NIH/NICHD grant R01 HD090071. SBS was supported by NIH/NICHD grant P50 HD028138 and the Robert and Laura Reynolds MGH Research Scholar Program. MFL was supported by NIH/National Institute of Diabetes and Digestive and Kidney Diseases grant T32 DK007028, a Postdoctoral Fellowship Award for Clinical Research from the MGH Executive Committee on Research Fund for Medical Discovery, and a Catalyst Medical Research Investigator Training Award from the Harvard Catalyst. This work was conducted with support from Harvard Catalyst I The Harvard Clinical and Translational Science Center (National Center for Research Resources and National Center for Advancing Translational Sciences/NIH award UL1 TR001102) and financial contributions from Harvard University and its affiliated academic healthcare centers. The content is solely the responsibility of the authors and does not necessarily represent the official views of Harvard Catalyst, Harvard University and its affiliated academic healthcare centers, or the NIH.

Address correspondence to: Yee-Ming Chan, 300 Longwood Avenue, Boston, Massachusetts 02115, USA. Phone: 617.355.2156; Email: Yee-Ming.Chan@childrens.harvard.edu. Or to: Stephanie B. Seminara, 55 Fruit Street, Boston, Massachusetts 02114, USA. Phone: 617.726.3038; Email: Stephanie. Seminara@mgh.harvard.edu. 
1. Avendaño MS, Vazquez MJ, Tena-Sempere M. Disentangling puberty: novel neuroendocrine pathways and mechanisms for the control of mammalian puberty. Hum Reprod Update. 2017;23(6):737-763.

2. Dhillo WS, et al. Kisspeptin-54 stimulates the hypothalamic-pituitary gonadal axis in human males. J Clin Endocrinol Metab. 2005;90(12):6609-6615.

3. Chan YM, et al. Kisspeptin resets the hypothalamic GnRH clock in men. J Clin Endocrinol Metab. 2011;96(6):E908-E915.

4. George JT, et al. Kisspeptin-10 is a potent stimulator of LH and increases pulse frequency in men. J Clin Endocrinol Metab. 2011;96(8):E1228-E1236.

5. Dhillo WS, et al. Kisspeptin-54 stimulates gonadotropin release most potently during the preovulatory phase of the menstrual cycle in women. J Clin Endocrinol Metab. 2007;92(10):3958-3966.

6. Jayasena CN, et al. The effects of kisspeptin-10 on reproductive hormone release show sexual dimorphism in humans. JClin Endocrinol Metab. 2011;96(12):E1963-E1972.

7. Chan YM, Butler JP, Sidhoum VF, Pinnell NE, Seminara SB. Kisspeptin administration to women: a window into endogenous kisspeptin secretion and GnRH responsiveness across the menstrual cycle. J Clin Endocrinol Metab. 2012;97(8):E1458-E1467.

8. George JT, Anderson RA, Millar RP. Kisspeptin-10 stimulation of gonadotrophin secretion in women is modulated by sex steroid feedback. Hum Reprod. 2012;27(12):3552-3559.

9. Chan YM, et al. Exogenous kisspeptin administration as a probe of GnRH neuronal function in patients with idiopathic hypogonadotropic hypogonadism. J Clin Endocrinol Metab. 2014;99(12):E2762-E2771.

10. Lippincott MF, Chan YM, Delaney A, Rivera-Morales D, Butler JP, Seminara SB. Kisspeptin Responsiveness Signals Emergence of Reproductive Endocrine Activity: Implications for Human Puberty. J Clin Endocrinol Metab. 2016;101(8):3061-3069.

11. Palmert MR, Dunkel L. Clinical practice. Delayed puberty. N Engl J Med. 2012;366(5):443-453.

12. Harrington J, Palmert MR. Clinical review: Distinguishing constitutional delay of growth and puberty from isolated hypogonadotropic hypogonadism: critical appraisal of available diagnostic tests. J Clin Endocrinol Metab. 2012;97(9):3056-3067.

13. Wu FC, Butler GE, Kelnar CJ, Sellar RE. Patterns of pulsatile luteinizing hormone secretion before and during the onset of puberty in boys: a study using an immunoradiometric assay. J Clin Endocrinol Metab. 1990;70(3):629-637.

14. Wu FC, Butler GE, Kelnar CJ, Huhtaniemi I, Veldhuis JD. Ontogeny of pulsatile gonadotropin releasing hormone secretion from midchildhood, through puberty, to adulthood in the human male: a study using deconvolution analysis and an ultrasensitive immunofluorometric assay. J Clin Endocrinol Metab. 1996;81(5):1798-1805.

15. Pitteloud N, et al. The role of prior pubertal development, biochemical markers of testicular maturation, and genetics in elucidating the phenotypic heterogeneity of idiopathic hypogonadotropic hypogonadism. J Clin Endocrinol Metab. 2002;87(1):152-160.

16. Shaw ND, et al. Expanding the phenotype and genotype of female GnRH deficiency. J Clin Endocrinol Metab. 2011;96(3):E566-E576.

17. Guerriero KA, Keen KL, Millar RP, Terasawa E. Developmental changes in GnRH release in response to kisspeptin agonist and antagonist in female rhesus monkeys (Macaca mulatta): implication for the mechanism of puberty. Endocrinology. 2012;153(2):825-836

18. Varimo T, Miettinen PJ, Känsäkoski J, Raivio T, Hero M. Congenital hypogonadotropic hypogonadism, functional hypogonadotropism or constitutional delay of growth and puberty? An analysis of a large patient series from a single tertiary center. Hum Reprod. 2017;32(1):147-153.

19. Spratt DI, Carr DB, Merriam GR, Scully RE, Rao PN, Crowley WF. The spectrum of abnormal patterns of gonadotropin-releasing hormone secretion in men with idiopathic hypogonadotropic hypogonadism: clinical and laboratory correlations. J Clin Endocrinol Metab. 1987;64(2):283-291.

20. Sykiotis GP, et al. Congenital idiopathic hypogonadotropic hypogonadism: evidence of defects in the hypothalamus, pituitary, and testes. J Clin Endocrinol Metab. 2010;95(6):3019-3027.

21. Smith JT, et al. Differential regulation of KiSS-1 mRNA expression by sex steroids in the brain of the male mouse. Endocrinology. 2005;146(7):2976-2984.

22. Santen RJ, Bardin CW. Episodic luteinizing hormone secretion in man. Pulse analysis, clinical interpretation, physiologic mech anisms. J Clin Invest. 1973;52(10):2617-2628. 